\title{
Tratamiento supermicroquirúrgico del linfocele inguinal con linfedema intercurrente: Restauración de la fisiología linfática
}

\author{
Nicolás Pereira C. ${ }^{1,2}$, Tomás Gantz V. ${ }^{2}$, Matías Kufeke M. ${ }^{1,2}$ y Josefa Venegas N. ${ }^{2}$
}

Supermicrosurgical approach to inguinal lymphocele with lymphedema: Restoring the lymphatic physiology

Introduction: Lymphocele may occur after the disruption of lymphatic channels during a surgical procedure. After inguinal lymphadenectomy are very common, and many different approaches have been tried to prevent or minimize the formation of lymphoceles with inconsistent results. Clinical Case: We report a case of a female patient who presented with right recurrent inguinal lymphocele and lower limb lymphedema after lymph-node biopsy that was successfully treated with lymphatic supermicrosurgery restoring the lymph flow. Lower extremity had an excess volume of 7,03\% compared to the healthy contralateral limb. Preoperative study with magnetic resonance lymphangiography and indocyanine green lymphography were done to identify intraoperatively lymphocele afferent and distal lymphatic vessels to perform lymphovenous anastomosis (LVA). Three different afferent lymphatics were identified and total capsulectomy was performed. Three end-to- end supermicrosurgical LVA in the groin and one distal LVA on the leg were performed. The surgery was uneventful, and there were no postoperative complications. In the follow-up, no lymphocele was noticed and lymphedema had visibly reduced with a reduction of excess volume of $105.26 \%$. Inguinal lymphocele and lymphedema can be successfully treated with supermicrosurgery since it is a physiological approach to restore the lymphatic flow, in order to avoid lymphocele recurrence and to improve lymphedema symptoms.

Key words: lymphocele; lymphedema; supermicrosurgery; lymphovenous anastomosis; lymphography.

\section{Resumen}

Introducción: El linfocele es una patología que puede ocurrir por la disrupción linfática durante una cirugía, siendo frecuente luego de una linfadenectomía inguinal. Existen diversos enfrentamientos para prevenirlo o minimizarlo, sin embargo, los resultados son inconsistentes. Caso clínico: Reportamos el caso de una mujer, con linfocele recurrente en zona inguinal derecha y linfedema distal de la extremidad secundario a una biopsia ganglionar, tratado exitosamente mediante supermicrocirugía para restaurar el drenaje linfático. La extremidad inferior tenía un exceso de volumen de 7,03\%. Se realizaron estudios preoperatorios con linfografía por resonancia magnética y linfografía con verde de indocianina para identificar los vasos linfáticos y realizar anastomosis linfático-venosas (ALV). Se identificaron tres vasos linfáticos aferentes y se realizó una capsulectomía total. Se realizaron tres ALV término-terminales supermicroquirúrgicas en zona inguinal y una ALV distal en pierna. Durante seguimiento no hubo recidiva del linfocele, evidenciándose una reducción del exceso de volumen de la extremidad afectada de un 105,26\%. El linfocele inguinal y linfedema pueden ser tratados exitosamente mediante supermicrocirugía, restaurando el flujo linfático de manera fisiológica, evitando la recurrencia de linfocele y mejorando los síntomas del linfedema.

Palabras clave: linfocele; linfedema; supermicrocirugía; anastomosis linfático-venosa; linfografía.
Departamento de Cirugía Plástica, Clínica Las Condes. Santiago, Chile.

2Departamento de Cirugía Plástica y Quemados, Hospital del Trabajador. Santiago, Chile.

Recibido 2019-11-07 y aceptado 2020-02-13

Correspondencia a: Dr. Nicolás Pereira C. npereira@cinicalascondes.cl 


\section{Introducción}

El linfocele es una colección de líquido linfático claro, sin una reacción inflamatoria o granulomatosa en el sitio de filtración. Puede desarrollar una cavidad exudativa bajo una herida cicatrizada de tejidos blandos con o sin tabiques. Esta complicación puede ocurrir luego de una disrupción de canales linfáticos durante un procedimiento quirúrgico desde 3 a 8 semanas hasta 1 año después de la cirugía. Debido a que son autolimitados, la mayoría de los linfocele postquirúrgicos son asintomáticos, no diagnosticados y resueltos sin ningún tipo de tratamiento. Un 4\%-7\% de los linfoceles posquirúrgicos son sintomáticos debido a un trastorno de su autoabsorción. La presencia de aumento de la presión linfática, inflamación, infección, cuerpo extraño o tejido cicatricial asociado a un acceso inguinal previo aumenta la probabilidad de linfocele persistente ${ }^{1}$.

Los linfoceles ocurren con mayor frecuencia posterior a intervenciones quirúrgicas que involucran extremidad inferior, zona inguinal, linfadenectomías pélvicas o trasplante renal. Es muy común posterior a una linfadenectomía inguinal o ílio-inguinal, con una incidencia aproximada del $23 \%^{2}$. Se ha reportado que la tasa de incidencia de linfocele inguinal, posterior a una biopsia de linfonodo centinela varía entre $2 \%-24 \%$, aumentando a $87 \%$ en pacientes sometidos a una linfadenectomía pelviana e inguinal por cáncer anogenital ${ }^{3,4}$. Las complicaciones linfáticas de heridas inguinales posterior a procedimientos de revascularización arterial pueden traer potenciales complicaciones catastróficas, ocurriendo en un $1,8 \%$ a $5 \%$ de los casos $^{5,6}$.

Existen diferentes enfrentamientos para prevenir o minimizar la formación de linfocele con resultados inconsistentes. En las últimas décadas, algunos reportes han sugerido que el uso de azul patente para identificar fuga de vasos linfáticos pudiese ser una solución preventiva a este problema en una primera cirugía $^{7,8}$.

Los tratamientos convencionales conservadores incluyen principalmente aspiración y compresión, los que tienen una mínima invasividad, pero en muchos casos no son efectivos. Se ha propuesto, además, el uso de escleroterapia, utilizando diferentes agentes esclerosantes para la pelvis, incluso para linfoceles subcutáneos, como la bleomicina, OK432, polidocanol, tetradecil sulfato de sodio, acetato de sodio, sellantes de fibrina, etanol y tetraciclina ${ }^{9-11}$. Esta terapia es muy simple, pero los agentes actúan provocando una intensa inflamación en las paredes del linfocele y de los vasos linfáticos, causando fibrosis y obliteración de ambos. Algunos tratamientos quirúrgicos consisten en exponer el interior del linfocele y ligar el tejido subcutáneo donde debieran estar los vasos linfáticos con filtración ${ }^{12}$. En ambos casos, existe un alto riesgo que se desarrolle o agrave el linfedema producto que la función del drenaje linfático se ve interrumpida.

Recientemente se han propuesto abordajes quirúrgicos utilizando técnicas de microcirugía y supermicrocirugía. Estos consisten en la restitución del flujo linfático mediante la anastomosis de los vasos linfáticos dañados a otro vaso linfático o hacia una vena, reconstruyendo el drenaje linfático $^{13,14}$.

En el presente artículo, reportamos el caso de una paciente con linfocele recidivante y linfedema intercurrente que fue tratado exitosamente a través de supermicrocirugía linfática.

\section{Caso clínico}

Paciente de 67 años, sexo femenino, sometida a una biopsia de ganglio inguinal derecho luego de 2 meses de masa palpable. La patología no demostró malignidad, siendo una adenopatía granulomatosa necrotizante crónica. Inmediatamente posterior a la cirugía, presentó fuga de líquido por la herida que progresó a seroma. Se realizaron múltiples drenajes percutáneos y al mes posterior a la biopsia, se realizó un desbridamiento quirúrgico. Dos días después, recurrió el seroma y se evidenció edema en la extremidad inferior derecha. A los 2 meses, se realiza otro desbridamiento con capsulectomía total y puntos de capitonaje, asociado vendaje compresivo para reducir el espacio muerto, sin embargo, se obtuvo el mismo resultado. Ocho meses posteriores a la cirugía inicial, la paciente consulta con el autor (N.P) en la Unidad de Cirugía Linfática del Departamento de Cirugía Plástica de Clínica Las Condes con un linfocele inguinal y linfedema con fóvea ipsilateral de la extremidad inferior. Se realiza drenaje percutáneo del linfocele obteniendo $200 \mathrm{ml}$ de líquido claro amarillento. La medición de la circunferencia de las extremidades y el cálculo de volumen se realizaron con la fórmula de cono truncado demostrando un exceso de 395,98 $\mathrm{ml}$ o $7,03 \%$ de exceso de volumen comparada con la extremidad contralateral. (PEV: porcentaje de exceso de volumen $=$ volumen de la extremidad afectada-volumen de la extremidad contralateral no afectada/volumen de a extremidad contralateral no afectada) (Figura 1).

Como estudio preoperatorio, se inyectó $1 \mathrm{ml}$ de 


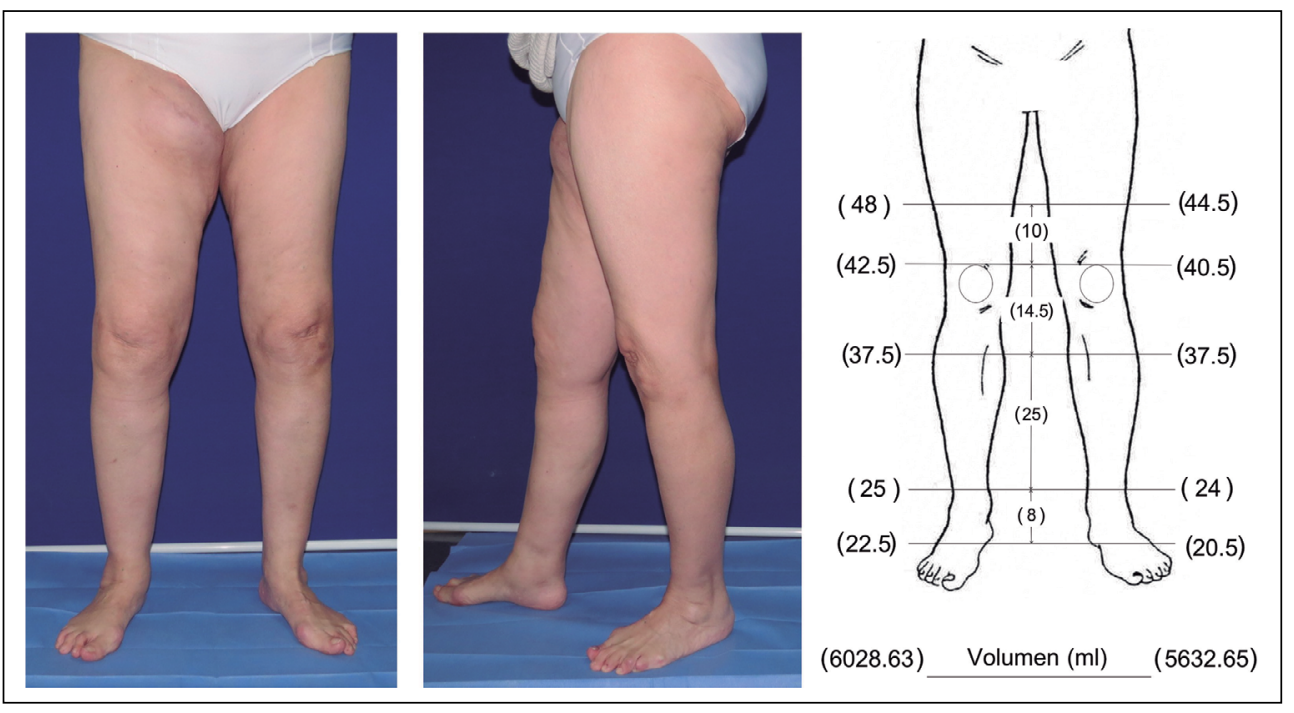

Figura 1. Mujer de 67 años con linfocele inguinal derecho y linfedema de extremidad inferior ipsilateral. Se evidencia un exceso de $395,98 \mathrm{ml}$ y un $7,03 \%$ de exceso de volumen, comparado con la extremidad sana contralateral. gadolinio en los espacios interdigitales y se realizó una linfangiografía por resonancia magnética (MR$\mathrm{L}$ ), evidenciándose la dilatación de canales linfáticos y un linfocele inguinal derecho (Figura 2A). Con estos hallazgos se mapearon los canales linfáticos para realizar las anastomosis linfático-venosas (LVA). Se realizó una linfografía con verde de indocianina preoperatoria para complementar el estudio e identificar de manera intraoperatoria los vasos linfáticos aferentes del linfocele.

Se realizó una incisión inguinal y exploración. Con el uso de un microscopio con fluorescencia cercana al infrarrojo (OPMI Pentero 800, Zeiss ${ }^{\circledR}$, Oberkochen, Alemania) se identificaron 3 vasos linfáticos aferentes con fuga de fluorescencia (Figura 2B) y se marcaron con un lápiz y una sutura nylon 6-0 (Figura 2C). Se realizó una capsulectomía total (Figura 2D) y se disecaron 3 vénulas. Se realizaron 3 LVA término-terminales con nylon 11-0 (Figura 2E) y una LVA distal en la pierna para contribuir con el tratamiento del linfedema.

No hubo complicaciones en el periodo postoperatorio y la paciente fue dada de alta en buenas condiciones, sin recurrencia del linfocele. A la semana, a las 2 semanas y un mes de seguimiento posterior a la supermicrocirugía, no se evidenció recurrencia linfocele. A los 8 meses posterior a la cirugía, el linfedema disminuyó considerablemente y el cálculo de volumen evidenció un exceso de $-21,37 \mathrm{ml}$ o un PEV de $-0,37 \%$ comparado con la extremidad sana contralateral, con una reducción del exceso de volumen de 105,26\% (REV: [PEV preoperatorio - PEV postoperatorio]/PEV preoperatorio) (Figura 3 ).
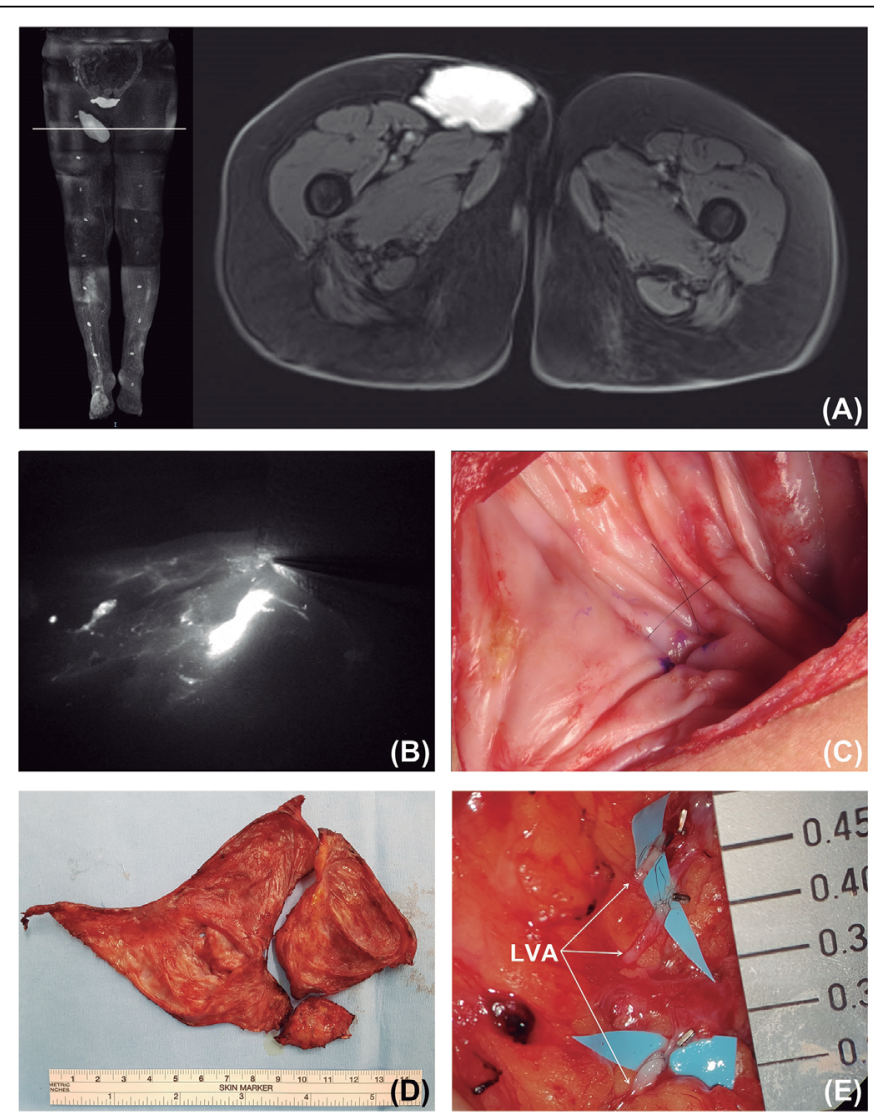

Figura 2. Resultados pre e intraoperatorios. A: Linfangiografía por resonancia magnética mostrando dilatación de canales linfáticos y un linfocele inguinal derecho. B: Fuga de fluido fluorescente (verde de indocianina) a través de vasos linfáticos aferentes lesionados, visualizados mediante un microscopio con fluorescencia cercana al infrarrojo. C: Marcación de vasos linfáticos aferentes con lápiz y nylon 6/0. D: Capsulectomía total. E: Tres LVA término-terminales supermicroquirúrgicas (LVA: anastomosis linfático-venosa). 


\section{CASOS CLÍNICOS}

Figura 3. Seguimiento de 8 meses. Reducción significativa de linfedema con un exceso de $-21,37 \mathrm{ml} \mathrm{y}-0,37 \%$ de volumen exceso de volumen comparado con la extremidad sana, con una reducción consecutiva del exceso de volumen de un $105,26 \%$. No se evidencia recurrencia del linfocele.

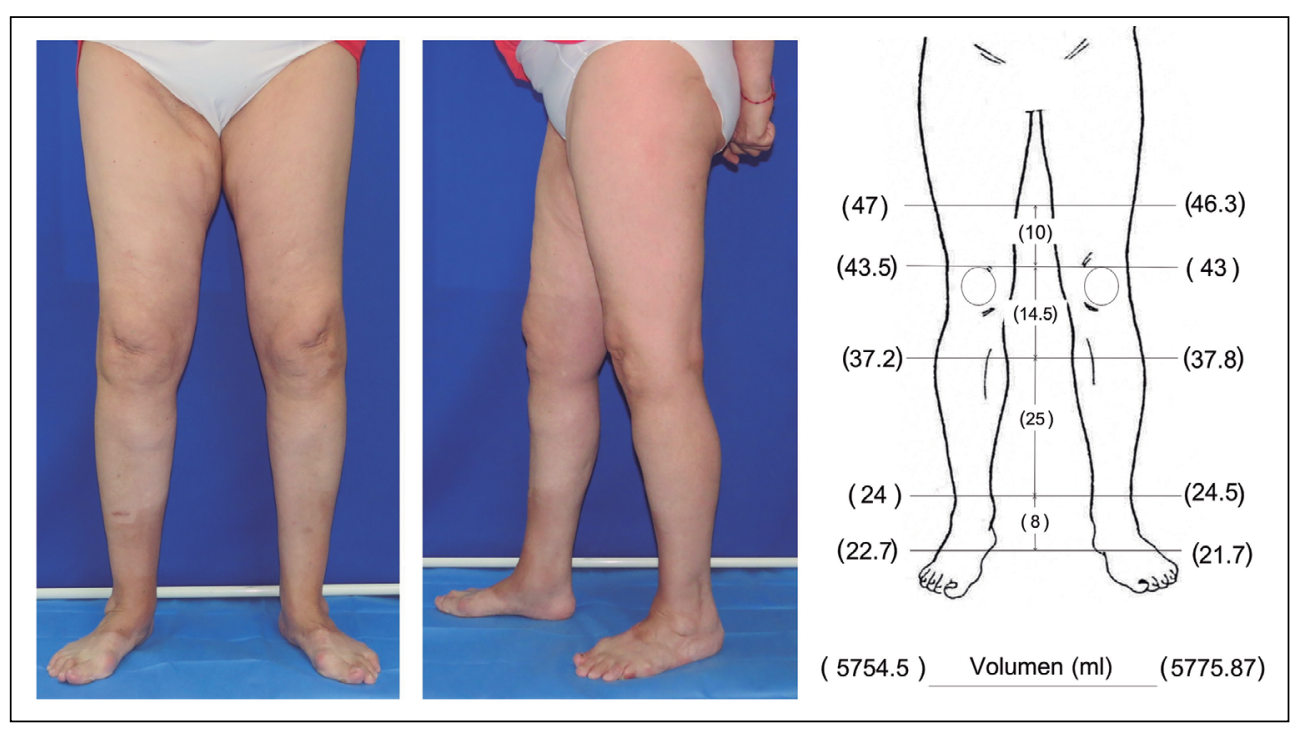

\section{Discusión}

El linfocele inguinal y linfedema intercurrente se pueden tratar exitosamente mediante supermicrocirugía, ya que es un tratamiento fisiológico para restaurar el flujo linfático, a fin de evitar la recurrencia del linfocele y mejorar los síntomas del linfedema.

Se han descrito diversos enfrentamientos conservadores. Se ha propuesto incluso el uso de la escleroterapia, y desde que Teruel y cols. ${ }^{9}$, reportaron el primer caso exitoso utilizando povidona yodada para el linfocele, se han utilizado muchos agentes esclerosantes para el tratamiento del linfocele subcutáneo y pelviano como la bleomicina, OK-432, polidocanol, tetradecil sulfato de sodio, acetato de sodio, sellantes de fibrina, etanol y tetraciclina ${ }^{10,11}$. Estos agentes actúan provocando una inflamación intensa de las paredes del linfocele y de los vasos linfáticos, causando fibrosis y obliteración de ambos.

Sansone y cols. ${ }^{15}$, propusieron una opción conservadora de compresión externa prolongada de la ingle mediante la aplicación de puntos de seda o prolene atadas a un pledget de plástico sobre el linfocele inguinal. Ellos reportaron resultados exitosos en la resolución del linfocele, sin restaurar la fisiología linfática.

Algunos tratamientos quirúrgicos consisten en la exploración del linfocele y la ligadura de vasos linfáticos guiados por azul patente. Toyserkani y cols. ${ }^{12}$, informaron una serie retrospectiva de 8 pacientes a los que se les realizó ligadura de sus vasos linfáticos con clips de titanio. Se evidenció una interrupción inmediata en la formación de linfocele en 6 pacientes con necesidad de punción en 2 pacientes, sin reportar la incidencia de linfedema en el seguimiento.

La ligadura de vasos linfáticos rotos tiene el riesgo de inducir linfedema distalmente y la inyección de esclerosantes implica un riesgo intrínseco de obliteración de los vasos linfáticos normales, incrementando el riesgo de linfedema postoperatorio de extremidades inferiores o empeoramiento de linfedema si ya es clínicamente evidente. El enfoque para restaurar el flujo linfático debe ser fisiológico, evitando y tratando el linfedema intercurrente. Boccardo y cols. ${ }^{13}$, propusieron un tratamiento microquirúrgico para el linfocele inguinal y el linfedema. Múltiples vasos linfáticos se anastomosaron a una rama de la vena safena mayor usando una técnica telescopada, logrando una curación completa del linfocele, sin aparición de linfedema postoperatorio en la extremidad inferior o una reducción considerable del volumen en casos donde el linfedema era sintomático.

Giacalone y cols. ${ }^{14}$, presentaron una serie de 4 pacientes con linfedema inguinal y parte superior de la pierna. Ellos realizaron LVA supermicroquirúrgicas desde la zona de fuga de los vasos linfáticos hasta una vena receptora de diámetro comparable. En nuestra opinión, consideramos que este es el enfoque más fisiológico, restaurando el flujo linfático y proporcionando un contacto intima-intimal de la anastomosis sin exposición de adventicia al lumen. A diferencia de lo que ellos informan respecto a la 
cápsula, nosotros creemos que es necesario realizar una capsulectomía total si nos vemos enfrentados a una cápsula gruesa y se debe realizar una evaluación del linfedema para saber si requerirá una LVA distal

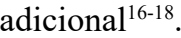

En resumen, el fracaso del tratamiento conservador y la recurrencia posterior al desbridamiento es común si no se restablece el flujo linfático. El tratamiento fisiológico supermicroquirúrgico para el linfocele inguinal tiene como objetivo restaurar el flujo linfático (Figura 4). La capsulotomía o capsulectomía debe evaluarse según la cronicidad y el grosor de la cápsula. Se debe preferir la realización de mapeos linfáticos con verde de indocianina para identificar los vasos linfáticos. Una vez identificados, el cabo linfático proximal o los vasos linfáticos intactos deben ser localizados para realizar las anastomosis linfático-linfáticas (LLA) terminoterminal o termino-lateral, respectivamente. Si no se localizan vasos linfáticos receptores, debe identificarse una vena receptora de diámetro similar para la realización de LVA. Finalmente, si hay edema concurrente en la extremidad inferior, se debe realizar una LVA distal para disminuir la presión en el sistema linfático y tratar el linfedema intercurrente.

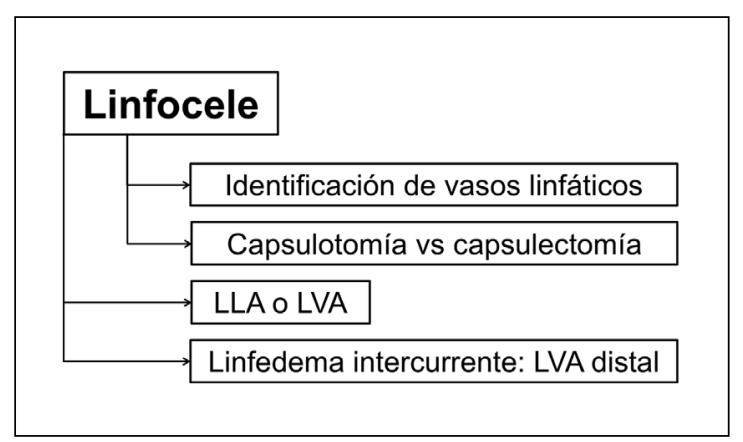

Figura 4. Enfrentamiento fisiológico supermicroquirúrgico para el tratamiento del linfocele inguinal (LLA: anastomosis linfáticolinfática; LVA: anastomosis linfático-venosa).

\section{Responsabilidades éticas}

Protección de personas y animales. Los autores declaran que para esta investigación no se han realizado experimentos en seres humanos ni en animales.

Confidencialidad de los datos. Los autores declaran que en este artículo no aparecen datos de pacientes.

Conflictos de interés: no hay.

\section{Bibliografía}

1. Shermak MA, Yee K, Wong L, Jones CE, Wong J. Surgical management of groin lymphatic complications after arterial bypass surgery. Plast Reconstr Surg. 2005;115:1954-62.

2. Söderman M, Thomsen JB, Sørensen JA. Complications following inguinal and ilioinguinal lymphadenectomies: A meta-analysis. J Plast Surg Hand Surg. 2016;50:315-20.

3. White I, Mills JK, Diggs B, Fortino HJ, Ellis MC, Vetto JT. Sentinel lymph node biopsy for melanoma: comparison of lymphocele rates by surgical technique. Am Surg. 2013;79:388-92.

4. Gotto GT, Yunis LH, Guillonneau B, Touijer K, Eastham JA, Scardino PT, et al. Predictors of symptomatic lymphocele after radical prostatectomy and bilateral pelvic lymph node dissection. Int J Urol. 2011;18:291-6.

5. Porcellini M, Iandoli R, Spinetti F, Bracale U, Di Lella D. Lymphoceles complicating arterial reconstruction of the lower limbs: outpatient conservative management. J Cardiovasc Surg. 2002;43:217-21.

6. Tyndall SH, Shepard AD, Wilczewski JM, Reddy DJ, Elliott JP Jr, Ernst CB. Groin lymphatic complications after arterial reconstruction. J Vasc Surg. 1994;19:85863.

7. Blana A, Denzinger S, Lenhart M, Wieland WF, Ganzer R. Treatment of a recurrent inguinal lymphocele in a penis cancer patient by lymphography and selective ligation of lymphatic vessels. Int J Urol. 2007;14:450-1.

8. Lavie O, Karmeli R, Mansano R, Hallak M, Bornstein J, Abramovici H. Treatment of recurrent inguinal lymphocele by lymphatic leakage mapping and subsequent ligation of lymphatic vessel endings: a case report. Gynecol Oncol. 2002;84:155-6.

9. Teruel JL, Escobar EM, Quereda C, Mayayo T. A simple and safe method for management of lymphocele after renal transplantation. J Urol. 1983;130:1058-9.

10. Clemens RK, Meier TO, Pfiffner R, Hafner J, Amann-Vesti B. Sclerotherapy of lymphatic cysts of the leg with OK432. Vasa 2012;41:149-53.

11. Klode J, Klötgen K, Körber A, Schadendorf D, Dissemond JJ. Polidocanol foam sclerotherapy is a new and effective treatment for post-operative lymphorrhea and lymphocele. Eur Acad Dermatol Venereol. 2010;24:904-9.

12. Toyserkani NM, Nielsen HT, Bakholdt V, Sørensen JA. Ligation of lymph vessels for the treatment of recurrent inguinal lymphoceles following lymphadenectomy. World J Surg Oncol. 2016;14:9-12.

13. Boccardo F, Dessalvi S, Campisi C, Molinari L, Spinaci S, Talamo G, et al. Microsurgery for groin lymphocele and lymphedema after oncologic surgery. Microsurgery 2014;34:10-3.

14. Giacalone G, Yamamoto T, Hayashi A, Belva F, Gysen M, Hayashi N, et al. Lymphatic supermicrosurgery for the treatment of recurrent lymphocele and severe lymphorrhea. Microsurgery 2019;39:326-31.

15. Sansone F, del Ponte S, Zingarelli E, Casabona R. The 'packing of the groin' technique: an innovative approach for 


\section{CASOS CLÍNICOS}

groin lymphocele. Interact Cardiovasc Thorac Surg. 2011;13:367-9.

16. Pereira N, Koshima I. Linfedema: actualización en el diagnóstico y tratamiento quirúrgico. Rev Chil Cir.
2018;70:589-97.

17. Pereira N, Yamamoto T. Linfedema de extremidades inferiores secundario al tratamiento oncológico: Actualización en el Diagnóstico y Tratamiento Quirúrgico.
Rev Cir. 2019;71:88-97.

18. Pereira N, Pons G, Masià J. Linfedema Asociado al Cáncer de Mama: Factores de Riesgo, Diagnóstico y Tratamiento Quirúrgico. Rev Cir. 2019;71:79-87. 